\title{
Extracting local symmetry of liquid metals from extended x-ray absorption fine structure using deep neural networks
}

\author{
F. lesari ${ }^{1}$, H. Setoyama ${ }^{2}$, T. Okajima ${ }^{1}$ \\ ${ }^{1}$ Aichi Synchrotron Radiation Center, 489-0965 Seto, Aichi, Japan, \\ ${ }^{2}$ Kyushu Synchrotron Light Research Center, 841-0005 Tosu, Saga, Japan \\ fabio.iesari@aichisr.jp
}

Due to its sensitivity to local structure, X-ray absorption spectroscopy is a powerful tool to study disordered systems. One of the most interesting property of XAFS is the sensitivity not only to pair distribution function, but also to three-body distribution, which contains information on bond angles between nearest neighbours. Reverse Monte Carlo (RMC) is a structural modelling method from which this information can be obtained [1], but it requires to know the density of the system being investigated, which may not be available especially in extreme thermodynamic conditions. Being a simulation method, it is also costly in terms of time. In recent years, neural networks (NN) have become a widely used tool to tackle different problems and have also been applied to the analysis of EXAFS data [2]. We wanted to investigate whether the same methodology could be applied to disordered systems and whether it would be possible to obtain information beyond the pair distribution function.

The critical point of any NN is the dataset used for the training process, that should be sufficiently large and heterogeneous. For this purpose, we ran several MD simulations of mono-atomic nickel at various temperatures for different crystal configurations, varying also the first-neighbour distance. The temperature was increased past the melting point to also include liquid configurations. From each configuration, we calculated the number distribution function, bond-angle distribution of the nearest neighbours and the EXAFS signal, using GNXAS suite of programs [3]. The created dataset was then used to optimize and train a set of deep NN to estimate number and bond-angle distribution from a given EXAFS signal.

We used the NN to analyse data of nickel at different temperatures and phases. Results from each NN are averaged and standard deviation calculated to estimate errors. Obtained results show that the $\mathrm{NN}$ is able to distinguish between ordered and disordered configurations and is also able to detect small changes in the local ordering of liquid structure, comparable with previously published results [4].

[1] Di Cicco A., Trapananti A., Faggioni S. \& Filipponi A. (2003). Phys. Rev. Lett. 91, 135505.

[2] Timoshenko J., Anspoks A., Cintins A., Kuzmin A., Purans J. \& Frenkel A. I. (2018). Phys. Rev. Lett. 120, 225502.

[3] Filipponi A. \& Di Cicco A. (1995). Phys. Rev. B 52, 15135.

[4] Di Cicco A., Iesari F., De Panfilis S., Celino M., Giusepponi S. \& Filipponi A. (2014). Phys. Rev. B 89, 060102.

\section{Keywords: EXAFS; liquids; neural networks}

This work is supported by JST CREST JPMJCR1861. 